OPEN ACCESS

Edited by:

Emilio Cusanelli,

University of Trento, Italy

Reviewed by:

Tahsin Stefan Barakat

Erasmus Medical Center, Netherlands

Ruslan Sadreyev,

Howard Hughes Medical Institute

(HHMI), United States

*Correspondence:

Hend M. El Tayeb

hend.saber@guc.edu.eg

Specialty section:

This article was submitted to

Cancer Genetics,

a section of the journal

Frontiers in Oncology

Received: 19 August 2019 Accepted: 05 December 2019

Published: 10 January 2020

Citation:

Salama EA, Adbeltawab RE and EI Tayebi HM (2020) XIST and TSIX: Novel Cancer Immune Biomarkers in

PD-L1-Overexpressing Breast Cancer Patients. Front. Oncol. 9:1459. doi: 10.3389/fonc.2019.01459

\section{XIST and TSIX: Novel Cancer Immune Biomarkers in PD-L1-Overexpressing Breast Cancer Patients}

\author{
Esraa A. Salama ${ }^{1}$, Reda E. Adbeltawab ${ }^{2}$ and Hend M. El Tayebi ${ }^{1 *}$ \\ ${ }^{1}$ Molecular Pharmacology Research Group, Department of Pharmacology and Toxicology, Faculty of Pharmacy and \\ Biotechnology, German University in Cairo, Cairo, Egypt, ${ }^{2}$ Department of Surgery, Faculty of Medicine, Ain Shams University, \\ Cairo, Egypt
}

Escaping antitumor immunity is a hallmark in cancer progression. Programmed cell death protein 1 (PD-1) is an immune checkpoint receptor responsible for the maintenance of immune tolerance; PD-1 ligand (PD-L1) is overexpressed in tumor cells, simplifying their escape from the immune system through T-cell function suppression. Notwithstanding that cancer antigen (CA)125, carcinoembryonic antigen (CEA), CA15-3, and alpha-fetoprotein (AFP) are among conventional breast cancer diagnostic biomarkers, their lack of sensitivity and specificity resides among their major limitations. Furthermore, human epidermal growth factor receptor (HER)2 and interleukin (IL)-6-demonstrated as breast cancer immune biomarkers-still possess limitations, for instance, technical detection problems and stability problems, which necessitate the discovery of novel, stable non-invasive cancer immune biomarkers. XIST and TSIX are two long non-coding (Inc)RNAs possessing a role in $\mathrm{X}$ chromosome inactivation $(\mathrm{XCl})$ as well as in breast cancer (BC). In the present study, they were investigated as stable non-invasive breast cancer immune biomarkers. The study demonstrated that PD-L1 was overexpressed in the different molecular subtypes of breast cancer patients as well as in MDA-MB-231 cells. Furthermore, IncRNAs XIST and TSIX were markedly increased in the tissues, lymph nodes, and different body fluids of breast cancer patients compared to controls. In addition, XIST and TSIX were differentially expressed in subtypes of BC patients, and their levels were correlated to PD-L1 expression level. In conclusion, this correlative study has shed light on the role of both IncRNAs XIST and TSIX as potential non-invasive $\mathrm{BC}$ immune biomarkers reflecting the evaded immune system of the patient and overcoming the instability problem of common BC biomarkers.

Keywords: non-invasive immune biomarkers, XIST, TSIX, PD-L1, liquid biopsies, IncRNAs

\section{INTRODUCTION}

Breast cancer $(\mathrm{BC})$ is the most commonly diagnosed cancer in females and has the highest fatality rate among other cancer types (1), competing with heart disease in being the leading cause of death worldwide (2). BC is classified into five subtypes: luminal A, luminal B, luminal B-like, human epidermal growth factor receptor (HER)2, and triple negative (3). The triple-negative BC (TNBC) subtype is the most aggressive subtype, and it is further classified into four subtypes: luminal/androgen receptor (LAR), mesenchymal (MES), basal-like immune suppressed (BLIS), and 
basal-like immune activated (BLIA) subtypes (4). The triplenegative subtype was demonstrated in several studies to frequently express the programmed cell death-ligand 1 (PDL1) $(5,6)$; there exist an overall immune system dysfunction and suppression throughout different BC subtypes due to the abundance of immune suppressive cells, namely, regulatory $\mathrm{T}$ cells (Tregs), and myeloid-derived suppressor cells (MDSCs), within the $\mathrm{BC}$ tumor microenvironment (7).

PD-L1 and its receptor programmed cell death protein 1 (PD-1) are immune checkpoint regulators promoting selftolerance by protecting the body from the excessive T-cell activity, inflammation, and autoimmunity (8). This is maintained through PD-1 (expressed on T cells)/PD-L1 (expressed mainly in nonlymphoid organs) ligand binding leading to T-cell suppression (9). In malignant state, the PD-1/PD-L1 signaling pathway is utilized as an immune escape mechanism for the tumor cells through the overexpression of PD-L1 by the cancer cells causing T-cell suppression and immune system escape (10). PD-L1 involvement in BC was highlighted in several studies. A recent study has postulated that TNBC tumors evade the immune system through upregulation of PD-L1 on the surface of TNBC cancer stem cells via the activation of WNT pathway (11). Another study demonstrated that PD-L1 expression in BC has been associated with grade 3 (G3), triple-negative subtype, and worse prognosis (12). Furthermore, the upregulation of PDL1 together with lactate dehydrogenase A (LDHA) in TNBC patients was related to poor patient outcome (5). Due to the involvement of the immune system in cancer progression, the emergence of immunotherapy represented a powerful weapon exclusively targeting dividing tumor cells as well as dispersed metastasis (13). The PD-L1 monoclonal antibody atezolizumab (14) and the eradication of TNBC through targeting glycosylated PD-L1 (15) signify successful examples of PD-L1 utilization in immunotherapy. Furthermore, cancer immune biomarker usage emerged from their ability to reflect patient immune status and his/her eligibility to immune therapy (16), in addition to the lack of sensitivity and specificity of conventional BC diagnostic biomarkers carcinoembryonic antigen (CEA), cancer antigen (CA)125, CA15-3, and alpha-fetoprotein (AFP) (17). Although, PD-L1 expression (18), high tumor mutation load (19), HER2 (20), and interleukin (IL)-6 (21) were successful illustrations of tumor and BC immune biomarkers, their technical detection problems (22) and instability in extended cryopreservation blood samples (23) have urged the discovery of novel stable immune biomarkers, the long non-coding (lnc)RNAs. To date, lncRNAs execute pivotal roles as cancer biomarkers as they are highly sensitive, specific, and stable in different body fluids, especially if they were circulating enclosed within apoptotic bodies or exosomes (24). It was reported in Shi et al. (25) that the IncRNAs successfully resisted ribonuclease enzyme as they were effectively detected in different body fluids where ribonuclease enzymes were present in rich quantities.

XIST and TSIX are two lncRNAs with a pivotal role in $\mathrm{X}$ chromosome inactivation (XCI) $(26,27)$ as well as in BC $(28$, 29). XCI is an important mechanism that compensates dosage disequilibrium introduced by the heteromorphic nature of the $\mathrm{X}$ and $\mathrm{Y}$ sex chromosomes in mammals so that only one $\mathrm{X}$ chromosome is transcriptionally active in both male and female cells. XCI is essential for the proper development and cellular differentiation as the presence of two active $\mathrm{X}$ chromosomes is correlated with a poorly differentiated state (30). In mice, XCI occurs in two waves. The initial wave commences in morula in preimplantation stage where lncRNA XIST expression is restricted only to inherited paternal silenced or imprinted (Xi) $\mathrm{X}$ chromosome. This is followed by lncRNA XIST expression suppression in all inner cell mass cells of blastocyst and $\mathrm{Xi}$ becomes reactivated. Finally, during embryo implantation, second wave of XCI initiates. This wave is characterized by random XCI where silencing of the $\mathrm{X}$ chromosome occurs through expression of IncRNA XIST in cis exclusively from future $\mathrm{X}$ inactive chromosome (Xi); this occurs through expression of lncRNA TSIX antisense to XIST, ensuring its repression from future active X chromosome (Xa) (31). In humans, XCI occurs similarly, yet with some discrepancies (31). Instead of TSIX gene, which has been marked in human genome, however, with no transcriptional evidence in human preimplant embryo, a different candidate lncRNA called $\mathrm{X}$ active coating transcript (XACT) was reported to be responsible for inhibition of XIST ability to silence X chromosome (31). To date, in humans, limited uncertain evidence for Xi status in cancer is accessible (32). It was reported in one study that in $\mathrm{BC}$, multiple $\mathrm{X}$ chromosomes (XXX or XXXX) were observed (33). However, another recent study postulated that the inactive $\mathrm{X}$ chromosome is epigenetically labile, which results in a double dose of $\mathrm{X}$ chromosome genes favoring cancer development (32). As for the contribution of lncRNAs XIST and TSIX in BC, their precise role is largely unknown with minimal studies reporting lncRNA XIST as a tumor suppressor lncRNA whose level is downregulated in BC $(28,29)$, with no suggestions about lncRNA TSIX role in BC. In this study, we aimed to investigate the role of lncRNAs XIST and TSIX as stable non-invasive BC immune biomarkers in different body fluids of BC patients and correlate their expression to PD-L1 expression.

\section{MATERIALS AND METHODS}

\section{Sample Collection}

BC biopsies, lymph nodes (LN), whole blood, serum, and nipple discharge (ND) were collected from 42 BC patients. It should be noted that nipple discharge collection was not available for all BC subtypes in this study. All BC patients were included based on the exclusion criteria (no other comorbidities other than BC). All samples were stored at $-80^{\circ} \mathrm{C}$ until further use. All subjects gave their written informed consent, and the Ain Shams University ethical review committee approved the study. The study followed the ethical guidelines of the 1975 Declaration of Helsinki. Peripheral blood mononuclear cells (PBMCs) were isolated from whole blood using Ficoll density gradient technique. Patients' clinical parameters are presented in Table 1 and Supplementary Table 1.

\section{Ficoll Density Gradient Technique}

Peripheral blood mononuclear cells (PBMCs) were isolated using Ficoll (Greiner Bio-One Ltd., Stonehouse, UK), as per the 
TABLE 1 | Patients characteristics.

\begin{tabular}{lc}
\hline Patients $(\boldsymbol{n}=\mathbf{4 2})$ & Percentage \\
\hline Gender & \\
Female 40/42 & $95 \%$ \\
Male 2/42 & $5 \%$ \\
Age & \\
Lower than 50 years old (15/42) & $35.7 \%$ \\
More than 50 years old (27/42) & $64.2 \%$ \\
Family history & \\
Positive family history (10/42) & $23.8 \%$ \\
Negative family history (32/42) & $76.19 \%$ \\
ER & \\
ER + VE (30/42) & $71.42 \%$ \\
ER - VE (12/42) & $28.57 \%$ \\
PR & \\
PR + VE 30/42 & $71.42 \%$ \\
PR - VE 12/42 & $28.57 \%$ \\
HER2/NEU & \\
HER2 + VE 8/42 & $19 \%$ \\
HER2 - VE 34/42 & $80 \%$ \\
LN metastasis & \\
LN + VE (24/42) & \\
LN - VE (18/42) & $57.14 \%$ \\
\hline
\end{tabular}

manufacturer's instructions. Harvested cells were washed twice in phosphate buffer saline (PBS, Applied Biosystems; Thermo Fisher Scientific Inc., cat. no. 10010023), and viable cells were counted using a hemocytometer. Cells were frozen in liquid nitrogen at a density of $10^{7} \mathrm{cells} / \mathrm{ml}$ in $90 \% \mathrm{v} / \mathrm{v}$ fetal bovine serum (FBS, Applied Biosystems; Thermo Fisher Scientific Inc., cat. no. 10270098) and 10\% v/v DMSO (Applied Biosystems; Thermo Fisher Scientific Inc., cat. no. D12345) for later analysis.

\section{Cell Culture}

MDA-MB 231 cells were purchased from Vacsera Egypt. They were incubated in Dulbecco's modified Eagle's medium (DMEM, Lonza, Germany, cat. no. 12-604F) supplemented with $4.5 \mathrm{~g} / \mathrm{L}$ glucose, $4 \mathrm{mmol} / \mathrm{L} \mathrm{L-glutamine,} \mathrm{10 \%} \mathrm{FBS} \mathrm{(Applied} \mathrm{Biosystems;}$ Thermo Fisher Scientific Inc., cat. no. 10270098), and MycoZap (1:500; Lonza, cat. no. LT07-818) at $37^{\circ} \mathrm{C}$ with an atmosphere of $5 \% \mathrm{CO}_{2}$ and $95 \%$ humidity. The cultured cells were then screened for PD-L1 expression.

\section{Transfection}

Twenty-four hours prior to transfection, seeding of $1-5 \times 10^{4}$ or $2-8 \times 10^{4}$ MDA-MB-231 cells (40-80\% confluency) per well of a 96-well plate or 24-well plate, respectively, was performed. The cells were incubated under normal growth conditions $\left(37^{\circ} \mathrm{C}\right.$ and 5\% $\mathrm{CO}_{2}$ ). MDA-MB 231 cells were transfected with siRNAs for both lncRNA XIST (Hs_XIST_3 FlexiTube siRNA, Qiagen Germany, cat. no. SI03654483) and lncRNA TSIX (Hs_TSIX_7 FlexiTube siRNA, Qiagen Germany, cat. no. SI04708795), in addition to performing a negative control using scrambled small interfering RNAs (Scr-siRNAs) that were not targeting lncRNA
XIST or TSIX. All transfection experiments were performed in triplicate using HiPerfect Transfection Reagent (Qiagen Germany, cat. no. 301705) according to the manufacturer's instructions, and experiments were repeated three times. Cells that were only exposed to transfection reagent were designated as mock cells, cells transfected with lncRNA XIST siRNA were designated as lncRNA XIST silenced cells, and cells transfected with lncRNA TSIX siRNA were designated as lncRNA TSIX silenced cells. This was followed by RNA extraction, screening for lncRNAs XIST, TSIX, and PD-L1 expression, and finally comparison to MDA-MB 231 mock cells.

\section{RNA Isolation}

RNA was isolated from MDA-MB231 cells, tumor tissues, lymph nodes, PBMCs, serum, and nipple discharge using TRIzol ${ }^{\mathrm{TM}}$ LS Reagent (Applied Biosystems; Thermo Fisher Scientific Inc., cat. no. 10296010) extraction protocol.

\section{Quantified Real-Time Polymerase Chain Reaction (qRT-PCR)}

Total RNA extracted was reverse-transcribed into singlestranded cDNA using the high-capacity cDNA reverse transcription kit (Applied Biosystems; Thermo Fisher Scientific Inc., cat. no. 4368814). The relative expression of lncRNAs XIST, TSIX, and PD-L1, along with B actin (as a housekeeping gene for normalization), was quantified and amplified using TaqMan RT-quantitative polymerase chain reaction (qPCR; Applied Biosystems; Thermo Fisher Scientific Inc., Assay IDs: Hs01079824_m1, Hs03299334_s1, Hs00204257_m1, and Hs01060665_g1, respectively) on a StepOne ${ }^{\mathrm{TM}}$ Real-Time PCR instrument (Applied Biosystems; Thermo Fisher Scientific Inc.). For every sample, a reaction mix was prepared according to the manufacturer's instructions, and $4 \mu \mathrm{l}$ of the respective cDNA was added. The RT-qPCR run was performed in the standard mode, consisting of two stages: a first 10 -min stage at $95^{\circ} \mathrm{C}$ where the Taq-polymerase enzyme was activated, followed by a second stage of 40 amplification cycles $\left(15 \mathrm{~s}\right.$ at 95 and $60 \mathrm{~s}$ at $\left.60^{\circ} \mathrm{C}\right)$. Relative expression was calculated using the $2^{-\Delta \Delta \mathrm{CT}}$ method. All PCR reactions including controls were run in triplicate reactions.

\section{Statistical Analysis}

All data were expressed in relative quantitation (RQ). To compare between two different studied groups, Student's unpaired $t$-test was employed. Data were expressed as mean \pm standard error of the mean (SEM). A $p<0.05$ was considered statistically significant. ${ }^{* * * *} p<0.0001,{ }^{* * *} p<0.001,{ }^{* *} p<0.01$, and ${ }^{*} p<0.05$. Analysis was performed using the GraphPad Prism 7.02 software.

\section{RESULTS}

\section{Expression of PD-L1 in Tumor Tissues and MDA-MB 231 Cells}

The entire pool of BC patients as well as MDA-MB 231 cells analyzed displayed a significant upregulation of PD-L1 mRNA ( $p=0.0101$ and $p=0.0002$, respectively) compared to controls. The results verified the variation in PD-L1 expression across 


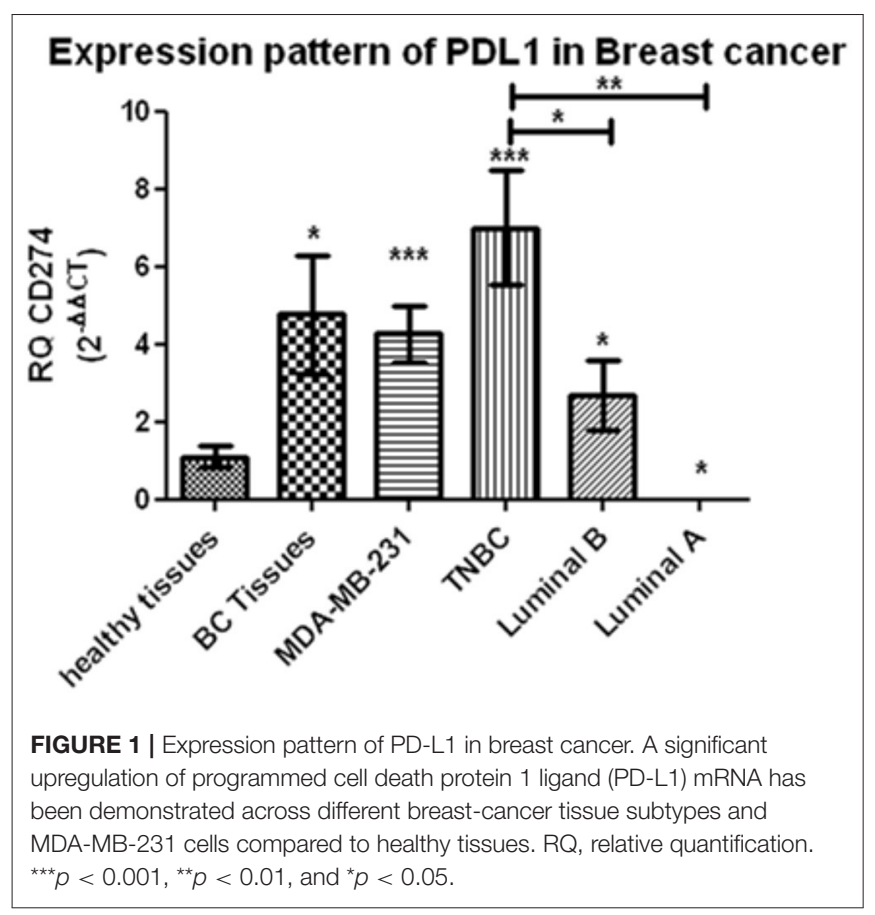

diverse $\mathrm{BC}$ patients' molecular subtypes as both luminal $\mathrm{B}$, and TNBC demonstrated significant upregulation of PD-L1 ( $p=0.0044$ and $p<0.0001$, respectively), in contrast to luminal A who displayed a significant downregulation of PDL1 $(p=0.0181)$, in addition to a non-significant expression of PD-L1 in HER2/luminal B and HER2/neu compared to controls. Moreover, TNBC exhibited a significant upregulation of PD-L1 compared to luminal B and luminal A samples $(p=0.0016$ and $p=0.0263$, respectively) (Figure 1).

\section{Expression Profiling of IncRNAs XIST and TSIX in Solid Body Sections From Different Molecular Subtypes of BC Patients}

Starting with BC tissues, there was a statistically significant upregulation in the expression of lncRNAs XIST and TSIX compared to healthy breast tissues $(p=0.0353$ and $p=0.0023$, respectively). Upon patient's stratification, both lncRNAs XIST and TSIX were significantly upregulated in luminal $\mathrm{A}$ and luminal B subtypes $(p=0.0341, p<0.0001, p=0.0400$, and $p=0.018$, respectively) compared to healthy breast tissues (Figures 2, 3, respectively). Furthermore, lncRNA XIST showed significant upregulation in HER2/neu subtype $(p=0.0344)$, although it demonstrated a non-significant change in expression in HER2/luminal B and TNBC subtypes compared to healthy breast tissues (Figure 2), in addition to significant upregulation of lncRNA TSIX in HER2/luminal B and TNBC subtypes ( $p<0.0001$, and $p<0.001$, respectively), although it demonstrated a non-significant change in expression in HER2/neu subtype compared to healthy breast tissues (Figure 3). To correlate lncRNAs XIST and TSIX expression in tissues to PD-L1 expression, patients were classified into high
(TNBC)-, medium (luminal B)-, and low (luminal A)-PD-L1expressing patients. IncRNA XIST demonstrated significant downregulation in high-PD-L1- compared to medium-PDL1-expressing patients $(p=0.0474)$. However, there was no significant difference in its expression between high- and low-PD-L1 patients. Additionally, lncRNA TSIX exhibited a significant upregulation in the high-compared to medium- and low-PD-L1-expressing patients $(p=0.0060$ and $p=0.0400$, respectively) (Figures 2, 3, respectively).

Proceeding to the lymph nodes, lncRNA XIST displayed a statistically significant upregulation in its expression $(p=0.0051)$, whereas there was a non-significant change in lncRNA TSIX expression in the combined BC patient's lymph nodes compared to non-metastatic lymph nodes. Additionally, lncRNAs XIST and TSIX displayed a significant upregulation in luminal A and luminal B subtypes $(p=0.0178, p<0.0001$, $p=0.0365$, and $p<0.0001$, respectively); nevertheless, they exhibited a non-significant change in expression in HER2/luminal B and TNBC, in addition to absent expression in HER2/neu subtype compared to non-metastatic lymph nodes. Besides, both lncRNAs XIST and TSIX demonstrated a significant downregulation in high compared to medium PD-L1 ( $p=0.0238$ and $p=0.0004$, respectively), and there was no significant difference in their expression between high- and low-PD-L1 patients (Figures 2, 3, respectively).

\section{Expression Profiling of IncRNAs XIST and TSIX in Body Fluids From Different Molecular Subtypes of BC Patients}

Starting with the serum, both lncRNAs XIST and TSIX displayed a statistically significant upregulation $(p=0.0007$ and $p=0.0363$, respectively) compared to controls. Additionally, they demonstrated a significant upregulation in luminal $\mathrm{A}$, luminal $\mathrm{B}$, and TNBC subtypes $(p=0.0003, p=0.0003$, $p<0.0001, p=0.0016, p<0.0001$, and $p=0.0315$, respectively) compared to controls (Figures 2, 3, respectively). Furthermore, lncRNA XIST exhibited a non-significant change in expression in HER2/luminal B and HER2/neu subtypes in contrast to lncRNA TSIX, which showed a significant upregulation in both subtypes compared to controls ( $p=0.0012$ and $p=0.0099$, respectively). Moreover, lncRNA XIST displayed significant upregulation in high-PD-L1 compared to patients expressing medium and low PD-L1 ( $p=0.0303$ and $p=0.0003$, respectively). As for lncRNA TSIX, it presented significant downregulation in high-compared to medium-PD-L1-expressing patients $(p=0.0047)$, yet there was no significant difference in its expression between high and low PD-L1 (Figures 2, 3, respectively).

There was a significant upregulation in the expression of lncRNAs XIST and TSIX in PBMCs of BC patients compared to controls ( $p=0.0499$ and $p=0.0242$, respectively). Additionally, they displayed a significant upregulation in luminal $\mathrm{A}$ and TNBC subtypes $(p=0.0063, p<0.0001, p=0.0026$, and $p<0.0001$, respectively), in addition to a non-significant change in their expression in HER2/luminal B and HER2/neu subtypes compared to controls. Furthermore, lncRNA XIST showed a statistical significant upregulation in luminal B 


\section{Expression Profiling of LncRNA XIST in subtypes of Breast Cancer}

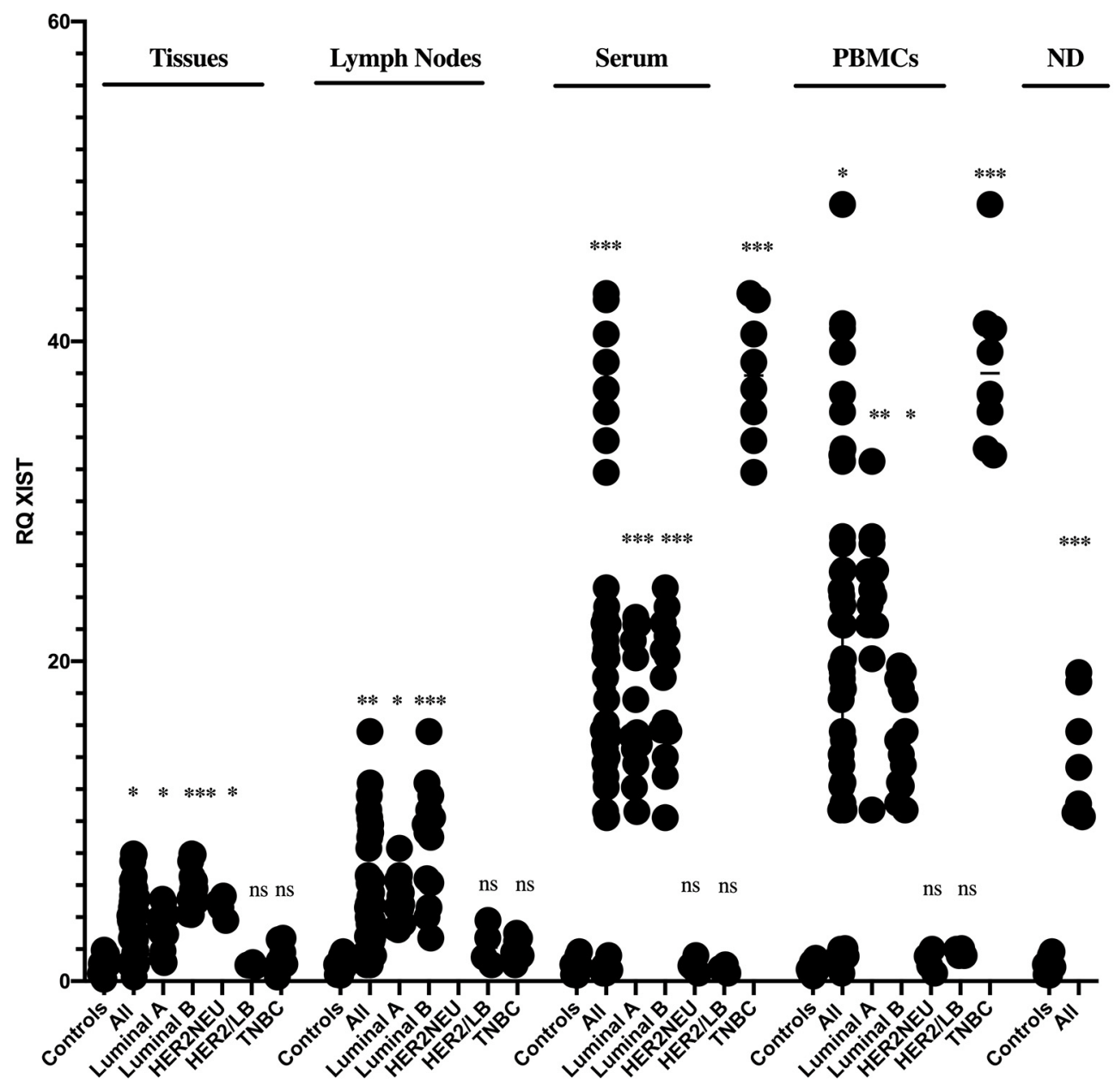

FIGURE 2 | Expression profiling of long non-coding (Inc)RNA XIST in breast cancer (BC) subtypes. Differential expression of IncRNA XIST has been investigated between each BC subtype and control (healthy sample) in addition to its differential expression through different programmed cell death protein 1 ligand (PD-L1)-expressing patients. IncRNA XIST showed a statistically significant downregulation in high-PD-L1-expressing compared to medium-PD-L1-expressing BC tissues and lymph nodes $(p=0.047$ and $p=0.0238$, respectively), in contrast to its statistically significant upregulation in high-PD-L1- compared to medium- and low-PD-L1-expressing BC serum and medium-PD-L1-expressing BC peripheral blood mononuclear cells (PBMCs) $(p=0.0303, p=0.0003$, and $p=0.0159$, respectively). High-PD-L1 patients denote triple-negative (TN)BCs. Medium PD-L1: luminal B and low PD-L1: luminal A. ND, nipple discharge; RQ, relative quantification. ${ }^{* \star *} p<0.001,{ }^{* \star} p<0.01$, and ${ }^{*} p<0.05$.

subtype $(p=0.00343)$ in contrast to TSIX lncRNA, which exhibited a non-significant change in expression in luminal $\mathrm{B}$ compared to controls. Finally, both lncRNAs exhibited significant upregulation in high- compared to medium-PD-L1-expressing patients ( $p=0.0159$ and $p=0.0175$, respectively). However, there was no significant difference in their expression between high- and low-PD-L1 patients (Figures 2, 3, respectively).

Finally, as for the nipple discharge, there was a statistically significant upregulation of both lncRNAs XIST and TSIX compared to controls $(p=0.0008$ and $p<0.0001$, respectively). Additionally, they displayed a significant upregulation in both luminal $B$ and TNBC subtypes compared to controls $(p<0.0001, p=0.0093, p=0.0008$, and $p<0.0001$, respectively). Furthermore, lncRNA XIST displayed significant downregulation in high- compared to
medium-PD-L1-expressing patients $(p=0.0284)$, in contrast to IncRNA TSIX, which showed a non-significant difference between high- and medium-PD-L1 patients (Figures 2, 3, respectively). Collectively, all these results evidenced that the pattern of expression of both lncRNAs XIST and TSIX in the solid body sections can be reflected in the different non-invasive body fluids of BC patients, thus confirming the role of XIST and TSIX lncRNAs as non-invasive immune biomarkers in BC patients.

\section{Expression of IncRNAs XIST and TSIX in Pre- and Post- menopausal BC Patients}

Extending the aim of this study to unraveling hormonal impact on both lncNRAs XIST and TSIX expression, BC patients were divided into pre- and postmenopausal groups. Both lncRNAs 


\section{Expression Profiling of LncRNA TSIX in Breast Cancer subtypes}

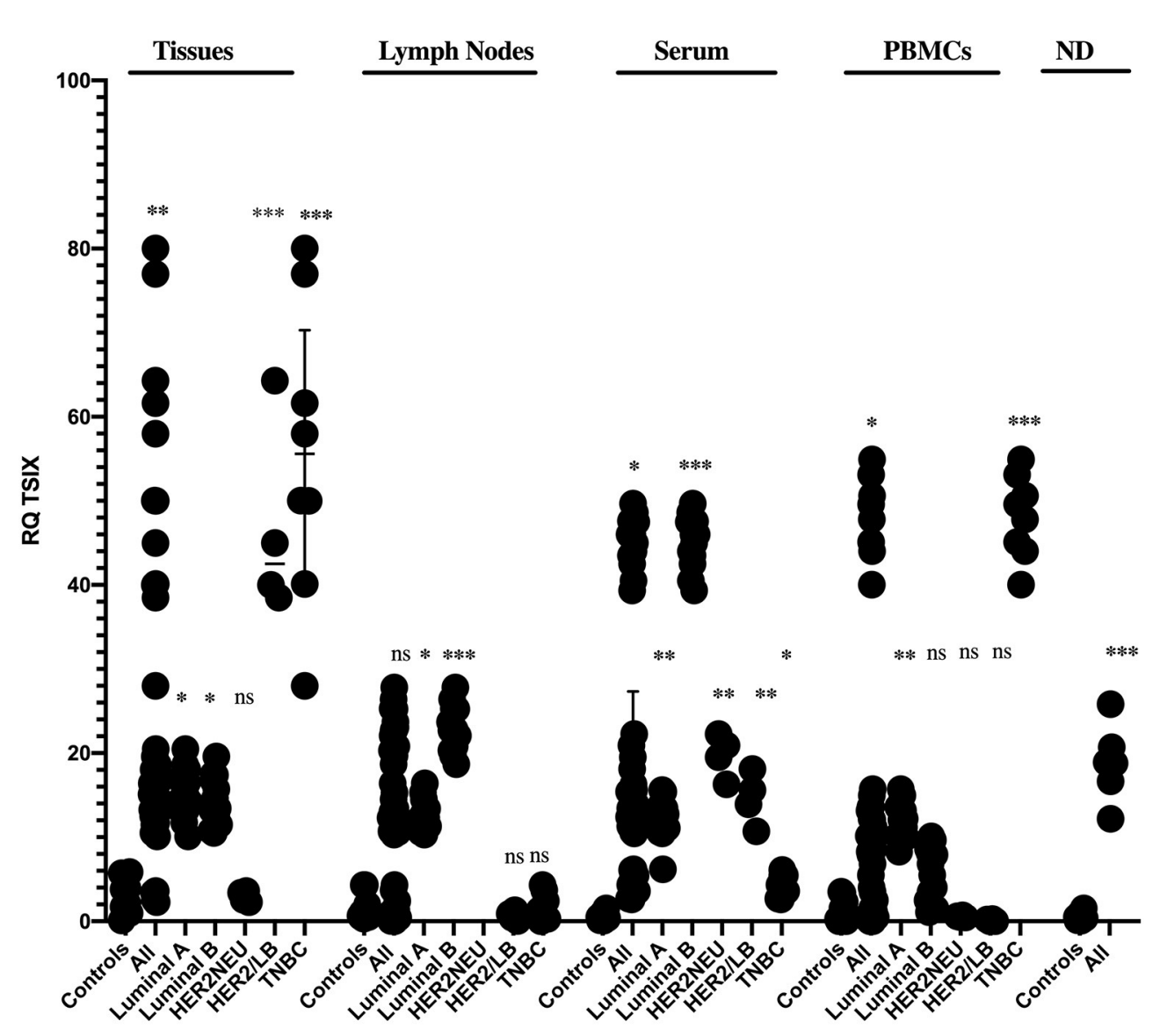

FIGURE 3 | Expression profiling of long non-coding (Inc)RNA TSIX in breast cancer (BC) subtypes. Differential expression of IncRNA TSIX has been analyzed between each BC subtype and control (healthy sample) in addition to its differential expression through different PD-L1-expressing patients. IncRNA TSIX displayed a statistically significant downregulation in high-PD-L1- compared to medium-PD-L1-expressing BC lymph nodes and serum $(p=0.0004$ and $p=0.0047$, respectively), in contrast to its statistical significant upregulation in high-PD-L1-compared to medium- and low-PD-L1-expressing BC tissues and medium-PD-L1-expressing BC PBMCs $(p=0.0060, p=0.0400$, and $p=0.0175$, respectively). High-PD-L1 patients denote triple-negative (TN)BCs. Medium PD-L1: luminal B and low PD-L1: luminal A. ND, nipple discharge; RQ, relative quantification. ${ }^{\star \star \star} p<0.001,{ }^{\star \star} p<0.01$, and ${ }^{*} p<0.05$.

XIST and TSIX displayed non-significant differences in their expression in the tissues, lymph nodes, and different body fluids of premenopausal BC patients compared to the postmenopausal BC patients (Figure 4), respectively, demonstrating that their level is unaffected by hormonal changes.

\section{Impact of IncRNAs XIST and TSIX Knockdown on PD-L1 mRNA Expression Level in MDA-MB-231 Cells}

Finally, to give weight to the correlation between lncRNAs XIST, TSIX, and PD-L1 expressions, the impact of lncRNAs XIST and TSIX in PD-L1 expression was investigated through their knockdown. Efficient knockdown of both lncRNAs XIST and TSIX in MDA-MB231 cells was confirmed $(p=0.0230$ and $p=0.0387$, respectively). As a consequence to $\operatorname{lncRNA}$
XIST repression, a dramatic elevation of PD-L1 was observed $(p<0.0001)$. However, a remarkable repression of PD-L1 was witnessed upon lncRNA TSIX repression $(p=0.0005)$ (Figure 5). There existed a non-significant difference between the mock and scrambled siRNA controls. For these data, an inverse relationship between XIST and PD-L1 has been observed, in contrast to a direct relationship between TSIX and PD-L1 expression.

\section{DISCUSSION}

While PD-1/PD-L1 pathway is pivotal to sustain an equilibrium between autoimmunity and peripheral tolerance, it impairs tumor immunity when employed by cancer cells promoting immune suppression and immune surveillance escape (34). For instance, PD-L1 overexpression in TNBC subtype establishes an immunosuppressive milieu through T-cell metabolic program 


\section{A premenopausal and postmenopausal BC patients}

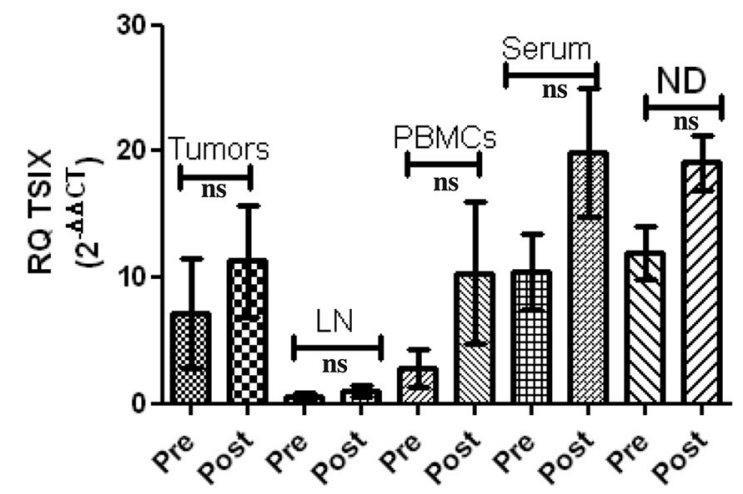

B

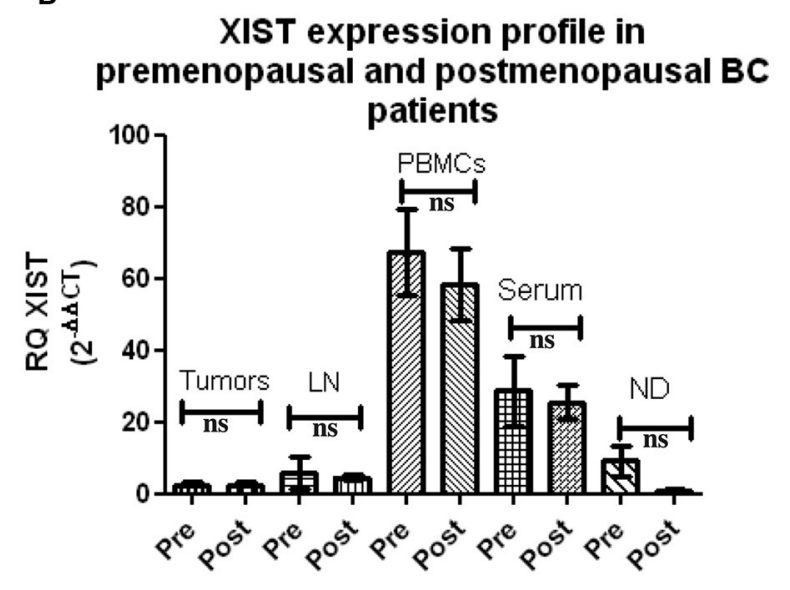

FIGURE 4 | Expression of long non-coding (Inc)RNAs XIST and TSIX in pre- and postmenopausal breast cancer (BC) patients. (A,B) Screening using qRT-PCR revealed a non-significant difference in the pattern of expression of both IncRNAs XIST and TSIX in solid body sections and different body fluids of pre- compared to postmenopausal $B C$ patients, respectively. ND, nipple discharge; $R Q$, relative quantification; ns, non-significant.

alteration, leading to impaired effector and memory T-cell differentiation and prevalence of immunosuppressive $\mathrm{T}$ cells (regulatory $\mathrm{T}$ cells) and exhausted $\mathrm{T}$ cells in peripheral blood of TNBC patients (Figure 6) $(34,35)$. Owing to the advances in the implementation of PD-L1 in BC immunotherapy $(14,15)$, the emergence of novel immune biomarkers that can surpass the limitations of current immune biomarkers is highly warranted $(22,23)$. lncRNAs represent ideal candidates as novel BC immune biomarkers due to their stability and their massive involvement in BC carcinogenesis $(24,25,36-38)$. A mounting body of evidence highlighted different contributing roles of the lncRNA XIST either as an oncogene or as a tumor suppressor gene in diverse cancers (39-43). Nonetheless, limited evidence displayed lncRNA XIST involvement as a tumor suppressor lncRNA in $\mathrm{BC}(28,29)$ in addition to the absence of concerns regarding lncRNA TSIX, the negative regulator of lncRNA XIST in BC. Furthermore, the correlation between XIST, TSIX, and PD-L1 so far is largely unexplored. This study was mainly focused on revealing the role of lncRNAs XIST and TSIX as potential stable non-invasive $\mathrm{BC}$ immune biomarkers in different liquid body fluids and correlating their expression to PD-L1 expression.

Thus, it was essential, first, to compare PD-L1 expression in different BC patient subtypes and TNBC cells to controls. TNBCs were found to have a significant high PD-L1 expression followed by luminal B and luminal A patients (Figure 1). And this is in accordance with previous studies that have put forward evidence that PD-L1 was significantly overexpressed in BC patients (44, 45). TNBC subtype will be referred to as high-PD-L1-expressing cells and luminal A and luminal B subtypes will be referred to as low-PD-L1-expressing cells.

Both lncRNAs XIST and TSIX expressions in tissues and for the first time lymph nodes of BC patients were compared to controls; our results displayed the significant upregulation of both lncRNAs XIST and TSIX in the BC patient tissues compared to healthy breast tissues. IncRNA XIST also displayed significant upregulation in malignant lymph nodes compared to benign lymph nodes (Figures 2, 3, respectively).

In harmony with our results, lncRNA XIST was proven to be upregulated in different types of cancer tissues compared to healthy tissues. For instance, the overexpressed lncRNA XIST in colorectal cancer promoted cell proliferation and invasiveness through controlling ZEB1 expression by acting as a ceRNA for miR-200b-3p (40). Upregulated lncRNA XIST in hepatocellular carcinoma was found, in another study, to activate AKT pathway through positive regulation of PDK1 expression via inhibiting miR-139-5p (41). However, other studies were not in line with our results and have proven the downregulation of lncRNA XIST in metastatic BC tissues. The downregulated lncRNA XIST enhanced BC tissue growth, migration, and invasiveness; upon upregulating lncRNA XIST BC tissues, migration and invasive capability were inhibited through miR-155/CDX1 axis (29). Another study reported that both lncRNAs XIST and TSIX were downregulated in BC tissues.

Downregulating lncRNA XIST in BC tissues improved the viability of $\mathrm{BC}$ cells through repressed expression of PHLPP1 (a phosphatase-inhibiting AKT phosphorylation) via increased HDAC3 recruitment to the PHLPP1 promoter, which had a mediatory effect on upregulation of AKT phosphorylation with no mention of any role for the downregulated TSIX (28). Additionally, upregulated lncRNA XIST in malignant lymph nodes was in alliance with further studies, postulating the candidate biomarker role of upregulated CD-169 (46), NUCB2 (47), and P-cadherin (48) in BC patients malignant lymph nodes. Furthermore, upon stratifying patients based on PD-L1 expression, high-PD-L1-expressing cells in BC tissues showed significant downregulation of lncRNA XIST and upregulation of lncRNA TSIX compared to low-PD-L1-expressing cells. In lymph nodes, high-PD-L1-expressing cells showed significant downregulation of both lncRNAs XIST and TSIX compared to low-PD-L1-expressing cells (Figure 7). 
A

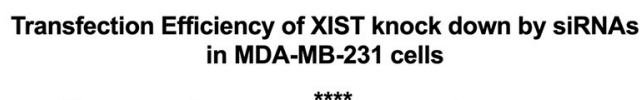

Transfection Efficiency of XIST knock down by siRNAs
in MDA-MB-231 cells

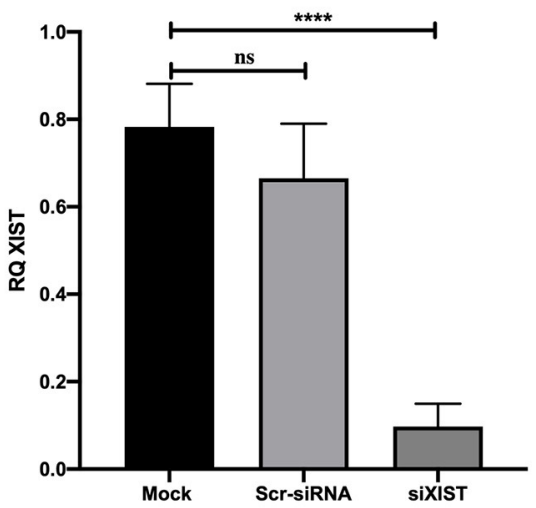

C

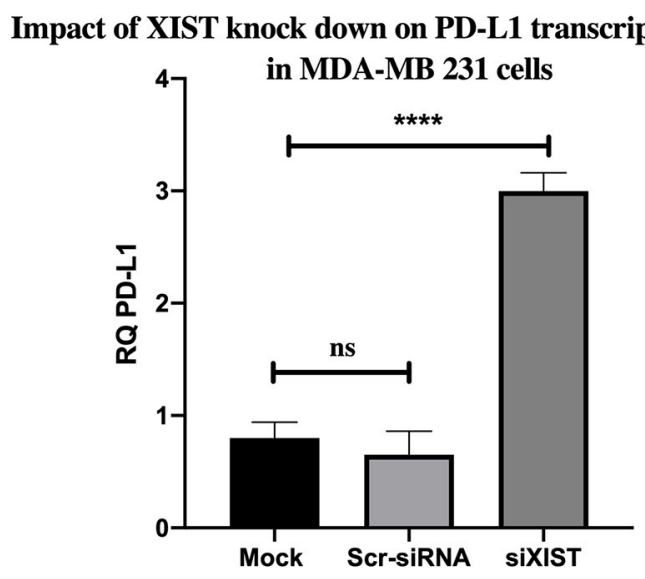

B

Transfection Efficiency of TSIX knock down by siRNAs

in MDA-MB-231 cells

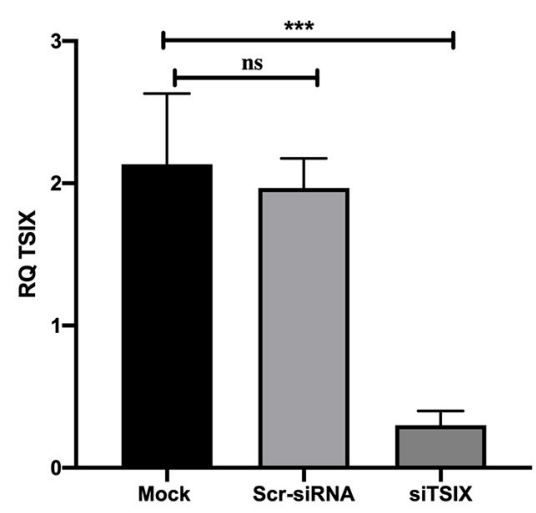

D

Impact of TSIX knock down on PD-L1 transcript expression in MDA-MB 231 cells

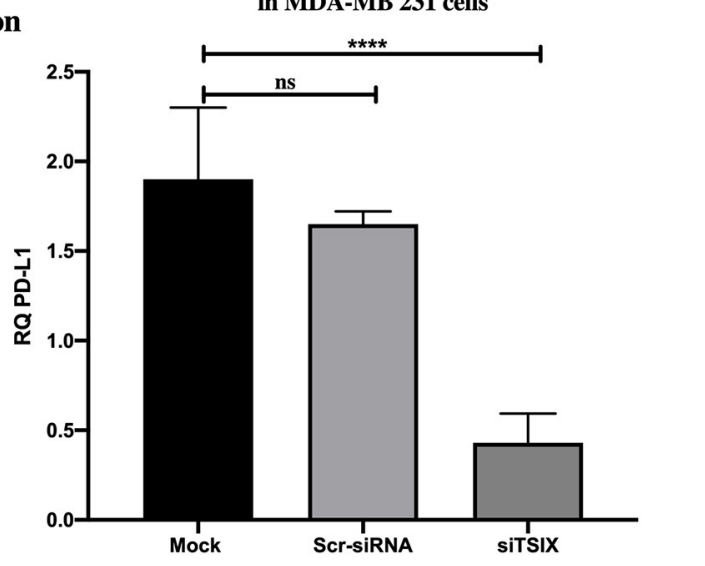

FIGURE 5 | Transfection efficiency of long non-coding (Inc)RNAs XIST and TSIX knockdown by siRNAs and the impact of their knockdown on programmed cell death protein 1 ligand (PD-L1) mRNA expression in MDA-MB-231 cells. (A,B) Confirmed efficient knockdown of IncRNAs XIST and TSIX compared to mock cells. (C,D) Knockdown impact of IncRNAs XIST and TSIX on PD-L1 expression. RQ, relative quantification. ${ }^{\star \star \star \star} p<0.0001 ;{ }^{* \star \star} p<0.001$.

As serum, nipple discharge, and PBMCs represent ideal noninvasive samples, it was mandatory to investigate whether they are capable of mirroring tissue and lymph node lncRNAs XIST and TSIX expression pattern and hence proving the lncRNAs XIST and TSIX role as potential stable non-invasive BC immune biomarkers. In this study, our data exhibited that both lncRNAs XIST and TSIX were significantly upregulated in the whole pool of BC serum, PBMCs, and nipple discharge compared to controls (Figures 2, 3, respectively). These results go in line with previous studies highlighting upregulated lncRNA XIST in non-small-cell lung cancer (49), lncRNA MALAT-1, IncRNA HOTAIR (50), miR-155 (51), and IL-6 (52) as upregulated serum $\mathrm{BC}$ patient diagnostic biomarkers. Moreover, our findings also go in line with previous studies that highlighted lncRNA XIST as an upregulated diagnostic biomarker in PBMCs of HCC patients (53) and miR-4484, miRK12-5-5p, and miR-3646 as upregulated biomarkers in nipple discharge of BC patients (54). In serum,
high-PD-L1-expressing cells revealed significant upregulation of lncRNA XIST and downregulation of lncRNA TSIX compared to low-PD-L1-expressing cells, while in PBMCs, high-PDL1-expressing cells showed significant upregulation of both lncRNAs XIST and TSIX compared to low-PD-L1-expressing cells, and finally, in nipple discharge, high-PD-L1-expressing cells demonstrated significant downregulation of lncRNA XIST compared to low-PD-L1-expressing cells (Figure 7).

To account for the discrepancy in lncRNA XIST expression in different body fluids, our hypothesis was that-based on the fact that ncRNAs expressed in the tissues can be released in a variety of body fluids $(55,56)$-the low lncRNA XIST expression in high-PD-L1-expressing nipple discharge cells is a result of its major release from tissues to serum rather than nipple discharge. An explanation for the high level of lncRNA XIST in high-expressing-PD-L1 PBMC cells can be explained by the fact that PBMCs have their own RNA machinery (57), 


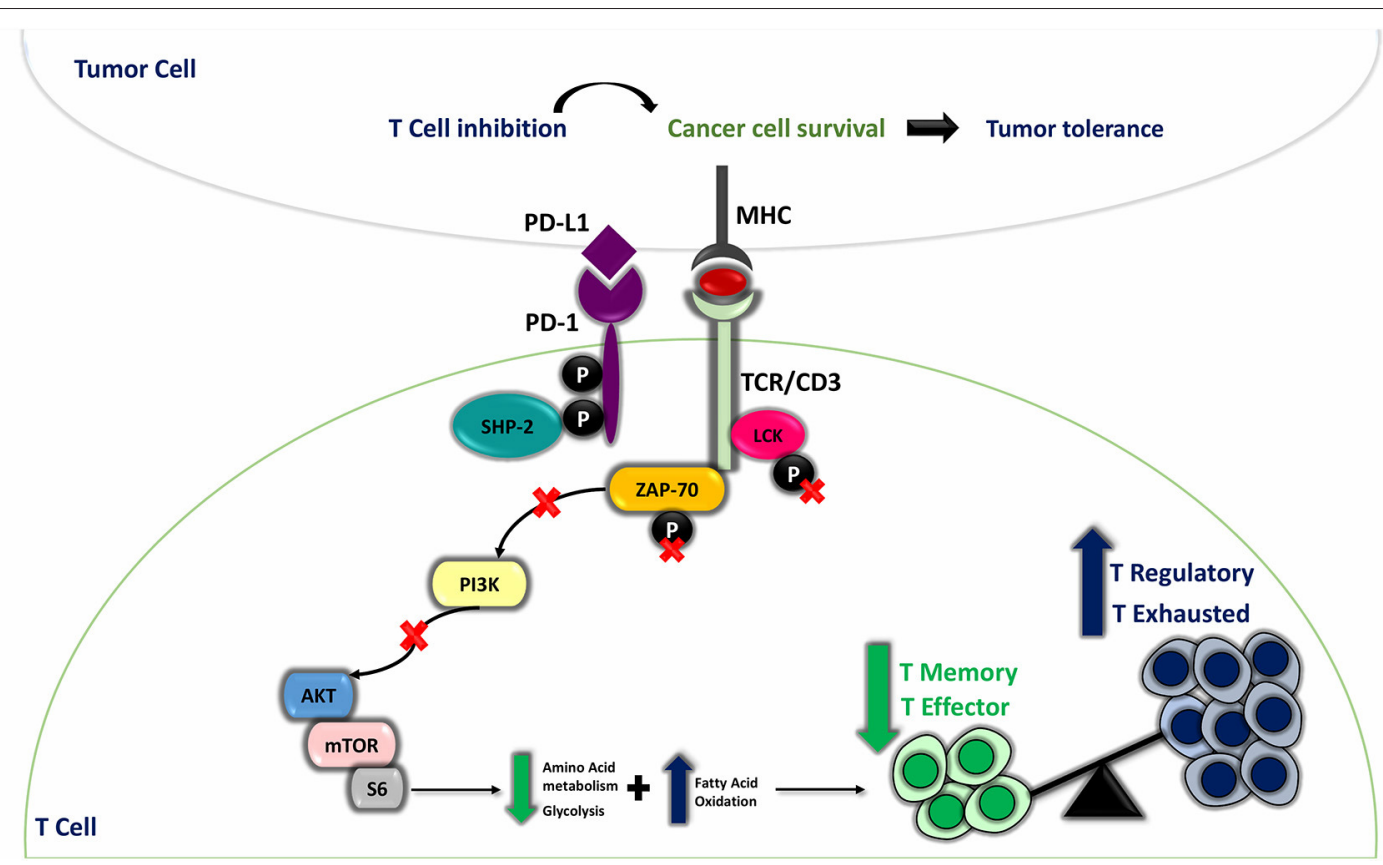

FIGURE 6 | Programmed cell death protein 1 (PD-1) ligand (PD-L1) involvement in T-cell mediated suppression and maintenance of immune evasion. Under physiological conditions, $T$ cell is activated against any foreign antigen presented on the surface of antigen-presenting cell through major histocompatibility complex $(\mathrm{MHC})$ by means of T-cell receptor (TCR) phosphorylation when oligomerization of TCR/CD3 chains takes place; this is followed by recruitment of activated Lck and Zap-70 to the phosphorylated immunoreceptor tyrosine-based activation motif (ITAM) of TCR tail, and this leads to the start of downstream TCR-signaling cascade. In cancer state, when PD-L1 present on cancer cells interacts with PD-1 expressed on T-cell surface, SHP-2 is recruited to ITSM, and the two tyrosine residues on PD-1 cytoplasmic tail acquire a phosphorylated status. This will result in dephosphorylated Lck and Zap-70 and hence their inactivation. Furthermore, PD-L1-PD-1 ligation suppresses PI3K/AKT/mTOR pathway, which affects normal T-cell metabolic pathway, shifting the pathway toward fatty acid oxidation (FAO) instead of glycolysis and amino acid metabolism, resulting in creation of immune suppressive milieu through enhanced T regulatory cells (immune suppressive cells) and exhausted T-cell differentiation and impaired effector and memory T cells differentiation.
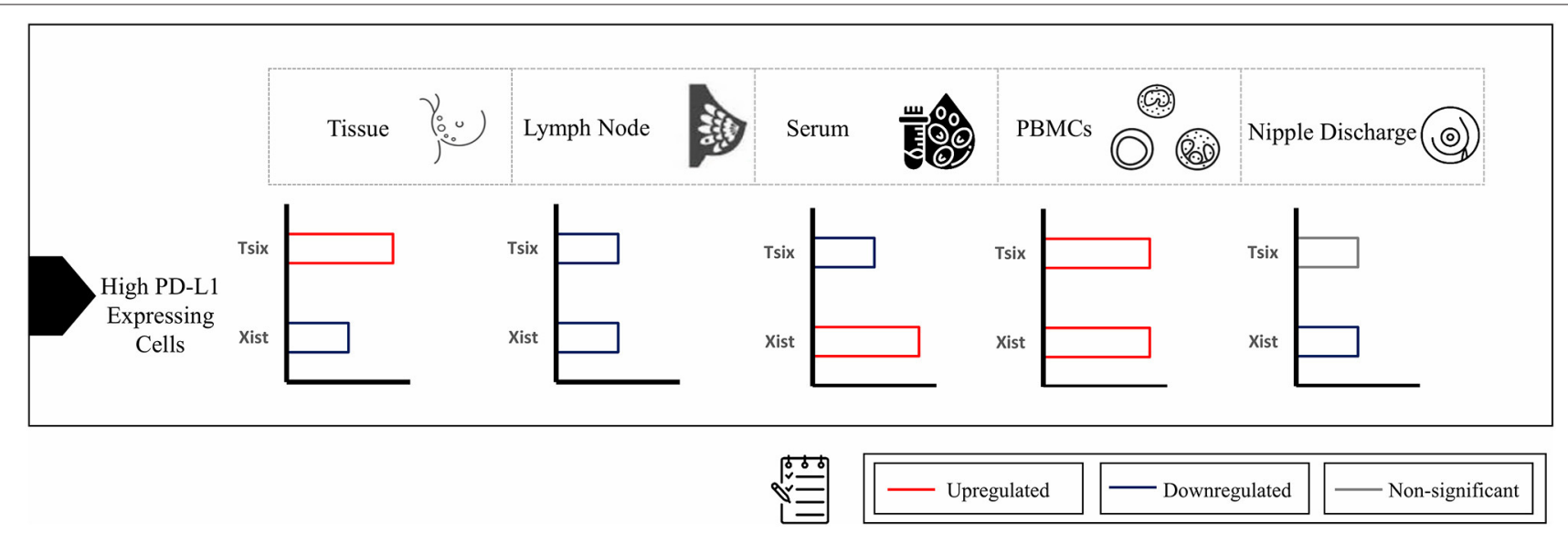

FIGURE 7 | Summary of expression of long non-coding (Inc)RNAs XIST and TSIX in high-programmed cell death protein 1 ligand (PD-L1)-expressing cells compared to low-PD-L1-expressing cells in different body parts. In high-PD-L1-expressing cells of breast-cancer tissues, there was significant downregulation of IncRNA XIST and upregulation of InCRNA TSIX compared to low-PD-L1-expressing cells; however, in lymph nodes, high-PD-L1-expressing cells showed significant downregulation of both IncRNAs XIST and TSIX compared to low-PD-L1-expressing cells. As for different body fluids investigated, the serum high-PD-L1-expressing cells displayed significant upregulation of IncRNA XIST and downregulation of IncRNA TSIX compared to low-PD-L1-expressing cells. In peripheral blood mononuclear cells (PBMCs), high-PD-L1-expressing cells demonstrated significant upregulation of both IncRNAs XIST and TSIX compared to low-PD-L1-expressing cells. Finally, in nipple discharge, high-PD-L1-expressing cells exhibited significant downregulation of IncRNA XIST compared to low-PD-L1-expressing cells.

which enables them to synthesize lncRNA XIST in the form of a counterregulatory response to combat immune evasion resulting from PD-L1 overexpression.
To prove XIST and TSIX lncRNAs hormonal independency, BC patients were subdivided into pre- and postmenopausal patients. The data reflected no significant difference between pre- 


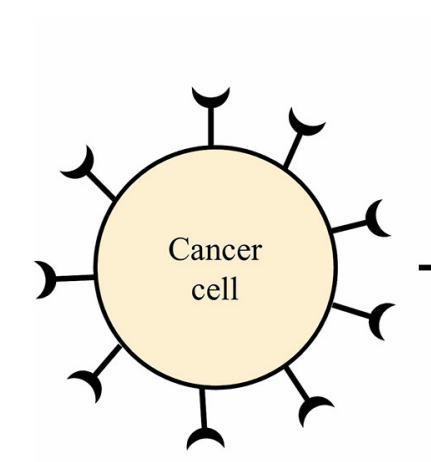

High PD-L1

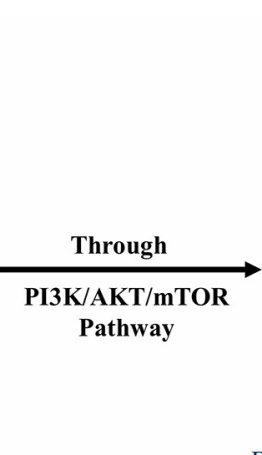

Directly

$\downarrow$

( $\downarrow$ XIST expression

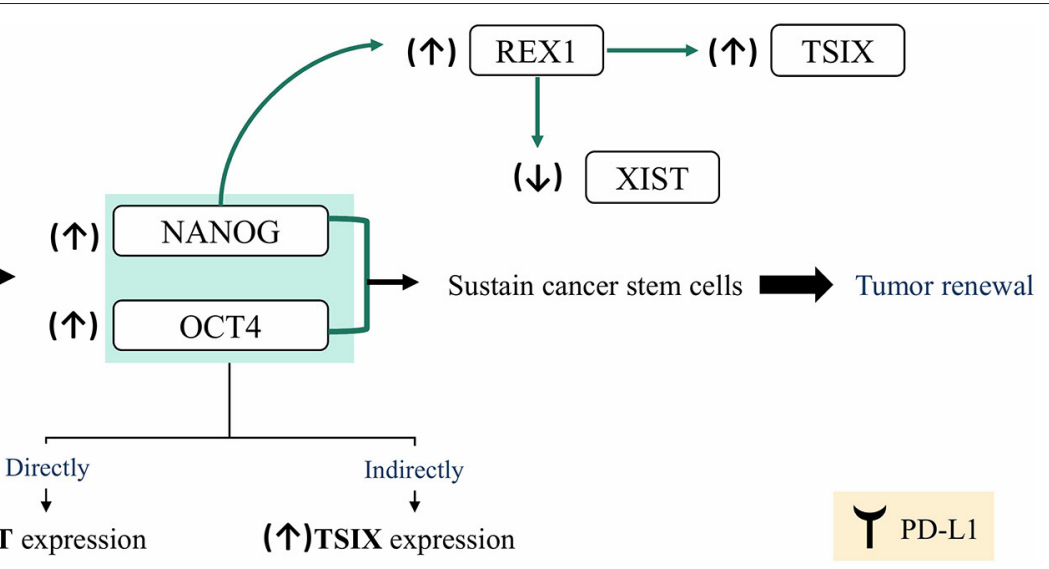

FIGURE 8 | Hypothesis of XIST/TSIX/PD-L1 regulation. In cancer cells, high- programmed cell death protein 1 ligand (PD-L1) expression sustains high level of both OCT4 and NANOG via PI3K/AKT/mTOR pathway activation, which results in sustained cancer cell stemness and tumor renewal. High level of OCT4 and Nanog will affect XIST and TSIX expression, resulting in repression of XIST expression and maintenance of high TSIX expression.

and postmenopausal women in terms of IncRNAs XIST and TSIX expression in different $\mathrm{BC}$ patient's body sections investigated (Figure 4). This is countered by other studies holding that some biomarkers are affected by hormonal changes such as serum level of vitamin $\mathrm{D}$, which was stated to be lowly expressed in perimenopausal women (58) and lncRNA HOTAIR, which was demonstrated to be dependent on estradiol level (59). Moreover, it should be noted that the postulation of pregnancy-induced protective effect against BC was not supported in our pool of patients as 85 and $15 \%$ of female patients were multiparous and nulliparous, respectively, in addition to two male patients, where all of the three groups had aggressive phenotypes of $\mathrm{BC}$ in our study. This is in opposition to a study indicating that early pregnancy reduces the risk of postmenopausal $\mathrm{BC}$ development in women through induction of chromatin remodeling in the breast of parous women compared to nulliparous women. This remodeling resides in a shift of nuclear state and appearance of cells forming lobules type 1 (lob 1 ) in parous breast to small and hyperchromatic nuclei with strong gene methylation and silencing, which is thought to be initiated by upregulated lncRNA XIST in parous breast compared to nulliparous women (60).

Finally, digging deeper to explore a correlation between lncRNAs XIST, TSIX, and PD-L1 expression in BC patients, TNBC cells (MDA-MB231 cells) were transfected with siRNAs for both lncRNAs XIST and TSIX followed by monitoring their knockdown impact on PD-L1 expression. The results disclosed a reverse association between PD-L1 and lncRNA XIST, where, upon lncRNA XIST knockdown, there was significant induction in PD-L1 expression, in contrast to lncRNA TSIX that significantly decreased PD-L1 expression upon its knockdown (Figure 5). This was consistent with previous reports in which lncRNA XIST was shown to act as a tumor suppressor in BC (28, 29) and with the fact that lncRNA TSIX is a negative regulator of IncRNA XIST (61).

To draw the full hypothesis regarding the mechanism by which XIST/TSIX/PD-L1 interacts, literature was screened.
Based on the literature review, it was evident that the recruitment of NANOG, OCT4, and REX1 has been implicated in the regulation of lncRNAs XIST and TSIX expression. One of the suggested mechanisms by which pluripotency factors NANOG and OCT4 directly repress IncRNA XIST expression is through binding to a region present within lncRNA XIST intron 1 (62), which was proven later to be dispensable for $\mathrm{X}$ chromosome inactivation (63), because deletion of intron 1 only mildly affects XCI as pluripotency factors act in concert toward inhibiting expression of XIST via binding to different sites present throughout the XIST (64) in addition to the indirect activation of lncRNA TSIX expression (64). REX1 recruitment is involved in direct repression of lncRNA XIST (65) as well as lncRNA TSIX activation and upregulation through binding to a regulatory region present in mice (called DPXas34) in lncRNA TSIX (66). Furthermore, NANOG positively regulates REX1 expression through binding to pluripotency-related transcription factor binding site located on REX1 promoter (67). Altogether, NANOG and OCT4 expression was correlated to PD-L1 expression and to the maintenance of $\mathrm{BC}$ stemness as highly expressed PD-L1 maintained expression of OCT4 and NANOG through PI3K/AKT/mTOR pathway activation in TNBC cells (68). Accordingly, XIST/TSIX/PD-L1 interaction resides in the high expression of PD-L1, which maintains NANOG and OCT4 expression; this will straightforwardly exert a repressing effect on lncRNA XIST expression and indirectly overexpresses lncRNA TSIX expression (Figure 8), yet this hypothesized interaction merits more detailed studies to ascertain its validity.

In addition to the uncertain mechanism of interaction between IncRNAs XIST, TSIX, and PD-L1, another limitation of this study is the unrevealed mechanistic contribution of both lncRNAs XIST and TSIX in BC progress as our main concern was exploring a correlation between lncRNAs XIST, TSIX, and PD-L1 expression in BC patients. Nevertheless, this study is strengthened by providing the first evidence of expression of both lncRNAs XIST and TSIX in lymph nodes, serum, PBMCs, and 
nipple discharge of $\mathrm{BC}$ patients correlated to $\mathrm{PD}-\mathrm{L} 1$ expression in addition to excluding their hormonal dependency and finally in shedding the light upon their significant non-invasive immune biomarker role that reflects the immune checkpoint (PD-L1) expression status in BC cells, which can be further utilized in the field of personalized medicine to ascertainwith no invasive means-the eligibility of $\mathrm{BC}$ patients for anti-PD-L1 treatment.

In conclusion, this study crystallizes the role of lncRNAs XIST and TSIX as stable non-invasive immune biomarkers better tolerated by patients compared to conventional biopsies with a potential role in reflecting the evaded immune status of BC patients.

\section{DATA AVAILABILITY STATEMENT}

All datasets generated for this study are included in the article/Supplementary Material.

\section{ETHICS STATEMENT}

The studies involving human participants were reviewed and approved by German University in Cairo and Ain Shams University Ethical Committees. The patients/participants

\section{REFERENCES}

1. Lin Y, Liu AY, Fan C, Zheng H, Li Y, Zhang C, et al. MicroRNA-33b inhibits breast cancer metastasis by targeting HMGA2, SALL4 and Twist1. Sci Rep. (2015) 5:1-12. doi: 10.1038/srep09995

2. McGee SF, Mazzarello S, Caudrelier JM, Lima MAG, Hutton B, Sienkiewicz $\mathrm{M}$, et al. Optimal sequence of adjuvant endocrine and radiation therapy in early-stage breast cancer - a systematic review. Cancer Treat Rev. (2018) 69:132-42. doi: 10.1016/j.ctrv.2018.06.015

3. Schnitt SJ. Classification and prognosis of invasive breast cancer: from morphology to molecular taxonomy. Mod Pathol. (2010) 23:60-4. doi: 10.1038/modpathol.2010.33

4. Burstein MD, Tsimelzon A, Poage GM, Covington KR, Contreras A, Fuqua SAW, et al. Comprehensive genomic analysis identifies novel subtypes and targets of triple-negative breast cancer. Clin Cancer Res. (2015) 21:168898. doi: 10.1158/1078-0432.CCR-14-0432

5. Huang X, Xie X, Wang H, Xiao X, Yang L, Tian Z, et al. PDL1 and LDHA act as ceRNAs in triple negative breast cancer by regulating miR-34a. J Exp Clin Cancer Res. (2017) 36:1-12. doi: 10.1186/s13046-017-0593-2

6. Sabatier R, Finetti P, Mamessier E, Adelaide J, Chaffanet M, Ali HR, et al. Prognostic and predictive value of PDL1 expression in breast cancer. Oncotarget. (2015) 6:5449-64. doi: 10.18632/oncotarget.3216

7. Khaja ASS, Toor SM, El Salhat H, Faour I, ul Haq N, Ali BR, et al. Preferential accumulation of regulatory $\mathrm{T}$ cells with highly immunosuppressive characteristics in breast tumor microenvironment. Oncotarget. (2017) 8:33159-71. doi: 10.18632/oncotarget.16565

8. Chakravarti N, Prieto VG. Predictive factors of activity of anti-programmed death-1/programmed death ligand-1 drugs: immunohistochemistry analysis. Transl Lung Cancer Res. (2015) 4:743-51. doi: 10.3978/j.issn.2218-6751.2015.12.10

9. Riley JL. PD-1 signaling in primary T cells. Immunol Rev. (2009) 229:11425. doi: 10.1111/j.1600-065X.2009.00767.x

10. Zou W, Chen L. Inhibitory B7-family molecules in the tumour microenvironment. Nat Rev Immunol. (2008) 8:467-77. doi: 10.1038/nri2326

11. Castagnoli L, Cancila V, Cordoba-Romero SL, Faraci S, Talarico G, Belmonte $\mathrm{B}$, et al. WNT signaling modulates PD-L1 expression in the stem cell provided their written informed consent to participate in this study.

\section{AUTHOR CONTRIBUTIONS}

ES has performed all the lab work and wrote the manuscript. $\mathrm{RA}$ is the clinical oncologist and surgeon who was responsible for providing all samples and clinical data. $\mathrm{HE}$ is the principal investigator and the main supervisor of this research work and has read and approved the final manuscript.

\section{ACKNOWLEDGMENTS}

The authors acknowledge the German University in Cairo for providing the required facilities to conduct this research work. Thanks are due to Mr. Muhammad Anwar, English instructor at the German University in Cairo, for the English-language editing.

\section{SUPPLEMENTARY MATERIAL}

The Supplementary Material for this article can be found online at: https://www.frontiersin.org/articles/10.3389/fonc. 2019.01459/full\#supplementary-material compartment of triple-negative breast cancer. Oncogene. (2019) 38:404760. doi: 10.1038/s41388-019-0700-2

12. Polónia A, Pinto R, Cameselle-Teijeiro JF, Schmitt FC, Paredes J. Prognostic value of stromal tumour infiltrating lymphocytes and programmed cell death-ligand 1 expression in breast cancer. J Clin Pathol. (2017) 70:8607. doi: 10.1136/jclinpath-2016-203990

13. Dimberu PM, Leonhardt RM. Cancer immunotherapy takes a multi-faceted approach to kick the immune system into gear. Yale J Biol Med. (2011) 84:37180. Retrieved from: http://medicine.yale.edu/yjbm

14. Schmid P, Adams S, Rugo HS, Schneeweiss A, Barrios CH, Iwata $\mathrm{H}$, et al. Atezolizumab and nab-paclitaxel in advanced triple-negative breast cancer. $N$ Engl J Med. (2018) 379:2108-21. doi: 10.1056/NEJMoa1809615

15. Li CW, Lim SO, Chung EM, Kim YS, Park AH, Yao J, et al. Eradication of triple-negative breast cancer cells by targeting glycosylated PD-L1. Cancer Cell. (2018) 33:187-201.e10. doi: 10.1016/j.ccell.2018.01.009

16. Gnjatic S, Bronte V, Brunet LR, Butler MO, Disis ML, Galon J, et al. Identifying baseline immune-related biomarkers to predict clinical outcome of immunotherapy. J Immunother Cancer. (2017) 5:1-18. doi: 10.1186/s40425-017-0243-4

17. Bolha L, Ravnik-Glavač M, Glavač D. Long noncoding RNAs as biomarkers in cancer. Dis Markers. (2017) 2017:7243968. doi: 10.1155/2017/7243968

18. Topalian SL, Hodi FS, Brahmer JR, Gettinger SN, Smith DC, McDermott DF, et al. Safety, activity, and immune correlates of anti-PD-1 antibody in cancer. $N$ Engl J Med. (2012) 366:2443-54. doi: 10.1056/NEJMoa 1200690

19. Alexandrov LB, Nik-Zainal S, Wedge DC, Aparicio SAJR, Behjati $\mathrm{S}$, Biankin $\mathrm{AV}$, et al. Signatures of mutational processes in human cancer. Nature. (2013) 500:415-21. doi: 10.1038/nature 12477

20. Kim JY, Lee E, Park K, Park WY, Jung HH, Ahn JS, et al. Immune signature of metastatic breast cancer: identifying predictive markers of immunotherapy response. Oncotarget. (2017) 8:47400-11. doi: 10.18632/oncotarget. 17653

21. Kil SH, Estephan R, Sanchez J, Zain JM, Kadin ME, Young JW, et al. PD-L1 is regulated by interferon gamma and interleukin 6 through STAT1 and STAT3 signaling in cutaneous T-cell lymphoma. Blood. (2017) 130:1458. 
22. Sapino A, Goia M, Recupero D, Marchiò C. Current challenges for HER2 testing in diagnostic pathology: state of the art and controversial issues. Front Oncol. (2013) 3:129. doi: 10.3389/fonc.2013.00129

23. Butterfield LH, Potter DM, Kirkwood JM. Multiplex serum biomarker assessments: technical and biostatistical issues. J Transl Med. (2011) 9:173. doi: 10.1186/1479-5876-9-173

24. Akers JC, Gonda D, Kim R, Carter BS, Chen CC. Biogenesis of extracellular vesicles (EV): exosomes, microvesicles, retrovirus-like vesicles, and apoptotic bodies. J Neurooncol. (2013) 113:1-11. doi: 10.1007/s11060-013-1084-8

25. Shi T, Gao G, Cao Y. Long noncoding RNAs as novel biomarkers have a promising future in cancer diagnostics. Dis Markers. (2016) 2016:9085195. doi: 10.1155/2016/9085195

26. Barakat TS, Gribnau J. X chromosome inactivation in the cycle of life. Development. (2012) 139:2085-9. doi: 10.1242/dev.069328

27. Stefan Barakat T, Gribnau J. X chromosome inactivation and embryonic stem cells. Adv Exp Med Biol. (2010) 695:13254. doi: 10.1007/978-1-4419-7037-4_10

28. Huang YS, Chang CC, Lee SS, Jou YS, Shih HM. Xist reduction in breast cancer upregulates AKT phosphorylation via HDAC3mediated repression of PHLPP1 expression. Oncotarget. (2016) 7:43256-66. doi: 10.18632/oncotarget.9673

29. Zheng R, Lin S, Guan L, Yuan H, Liu K, Liu C, et al. Long non-coding RNA XIST inhibited breast cancer cell growth, migration, and invasion via miR-155/CDX1 axis. Biochem Biophys Res Commun. (2018) 498:10028. doi: 10.1016/j.bbrc.2018.03.104

30. Vallot C, Ouimette JF, Rougeulle C. Establishment of X chromosome inactivation and epigenomic features of the inactive $\mathrm{X}$ depend on cellular contexts. BioEssays. (2016) 38:869-80. doi: 10.1002/bies.201600121

31. Sahakyan A, Plath K, Rougeulle C. Regulation of X-chromosome dosage compensation in human: mechanisms and model systems. Philos Trans R Soc B Biol Sci. (2017) 372:20160363. doi: 10.1098/rstb.2016.0363

32. Chaligné R, Heard E. X-chromosome inactivation in development and cancer. FEBS Lett. (2014) 588:2514-22. doi: 10.1016/j.febslet.2014.06.023

33. Wutz A, Valencia K. Recent insights into the regulation of X-chromosome inactivation. Adv Genomics Genet. (2015) 5:227. doi: 10.2147/AGG.S60399

34. Bardhan K, Anagnostou T, Boussiotis VA. The PD1: PD-L1/2 pathway from discovery to clinical implementation. Front Immunol. (2016) 7:550. doi: 10.3389/fimmu.2016.00550

35. Foulds GA, Vadakekolathu J, Abdel-Fatah TMA, Nagarajan D, Reeder $\mathrm{S}$, Johnson $\mathrm{C}$, et al. Immune-phenotyping and transcriptomic profiling of peripheral blood mononuclear cells from patients with breast cancer: identification of a 3 gene signature which predicts relapse of triple negative breast cancer. Front Immunol. (2018) 9:2028. doi: 10.3389/fimmu.2018.02028

36. Müller V, Oliveira-Ferrer L, Steinbach B, Pantel K, Schwarzenbach H. Interplay of lncRNA H19/miR-675 and lncRNA NEAT1/miR-204 in breast cancer. Mol Oncol. (2019) 13:1137-49. doi: 10.1002/1878-0261.12472

37. Gu J, Wang Y, Wang X, Zhou D, Shao C, Zhou M, et al. Downregulation of IncRNA GAS5 confers tamoxifen resistance by activating miR-222 in breast cancer. Cancer Lett. (2018) 434:1-10. doi: 10.1016/j.canlet.2018.06.039

38. Tang J, Li Y, Sang Y, Yu B, Lv D, Zhang W, et al. LncRNA PVT1 regulates triple-negative breast cancer through KLF5/beta-catenin signaling. Oncogene. (2018) 37:4723-34. doi: 10.1038/s41388-018-0310-4

39. Li C, Wan L, Liu Z, Xu G, Wang S, Su Z, et al. Long non-coding RNA XIST promotes TGF- $\beta$-induced epithelial-mesenchymal transition by regulating miR-367/141-ZEB2 axis in non-small-cell lung cancer. Cancer Lett. (2018) 418:185-95. doi: 10.1016/j.canlet.2018.01.036

40. Chen DL, Chen LZ, Lu YX, Zhang DS, Zeng ZL, Pan ZZ, et al. Long noncoding RNA XIST expedites metastasis and modulates epithelialmesenchymal transition in colorectal cancer. Cell Death Dis. (2017) 8:e3011. doi: 10.1038/cddis.2017.421

41. Mo Y, Lu Y, Wang P, Huang S, He L, Li D, et al. Long noncoding RNA XIST promotes cell growth by regulating miR-1395p/PDK1/AKT axis in hepatocellular carcinoma. Tumor Biol. (2017) 39:1010428317690999. doi: 10.1177/1010428317690999

42. Samir A, Salama E, El-Tayebi HM. The long non-coding RNA XIST: a new cornerstone in carcinogenesis. J Mol Genet Med. (2018) 12:116. doi: $10.4172 / 1747-0862.1000356$
43. Du Y, Weng XD, Wang L, Liu XH, Zhu HC, Guo J, et al. LncRNA XIST acts as a tumor suppressor in prostate cancer through sponging miR-23a to modulate RKIP expression. Oncotarget. (2017) 8:9435870. doi: 10.18632/oncotarget.21719

44. Mittendorf EA, Philips AV, Meric-Bernstam F, Qiao N, Wu Y, Harrington S, et al. PD-L1 expression in triple-negative breast cancer. Cancer Immunol Res. (2014) 2:361-70. doi: 10.1158/2326-6066.CIR-13-0127

45. Muenst S, Schaerli AR, Gao F, Däster S, Trella E, Droeser RA, et al. Expression of programmed death ligand 1 (PD-L1) is associated with poor prognosis in human breast cancer. Breast Cancer Res Treat. (2014) 146:1524. doi: 10.1007/s10549-014-2988-5

46. Shiota T, Miyasato Y, Ohnishi K, Yamamoto-Ibusuki M, Yamamoto $\mathrm{Y}$, Iwase $\mathrm{H}$, et al. The clinical significance of CD169-positive lymph node macrophage in patients with breast cancer. PLoS ONE. (2016) 11:e0166680. doi: 10.1371/journal.pone.0166680

47. Zeng L, Zhong J, He G, Li F, Li J, Zhou W, et al. Identification of nucleobindin-2 as a potential biomarker for breast cancer metastasis using iTRAQ-based quantitative proteomic analysis. J Cancer. (2017) 8:30629. doi: 10.7150/jca.19619

48. Vieira AF, DIonísio MR, Gomes M, Cameselle-Teijeiro JF, Lacerda M, Amendoeira I, et al. P-cadherin: a useful biomarker for axillary-based breast cancer decisions in the clinical practice. Mod Pathol. (2017) 30:698709. doi: 10.1038/modpathol.2016.232

49. Tantai J, Hu D, Yang Y, Geng J. Combined identification of long non-coding RNA XIST and HIF1A-AS1 in serum as an effective screening for non-small cell lung cancer. Int J Clin Exp Pathol. (2015) 8:7887-95. Retrieved from: http://www.ijcep.com/

50. Zhang L, Song X, Wang X, Xie Y, Wang Z, Xu Y, et al. Circulating DNA of HOTAIR in serum is a novel biomarker for breast cancer. Breast Cancer Res Treat. (2015) 152:199-208. doi: 10.1007/s10549-015-3431-2

51. Sun Y, Wang M, Lin G, Sun S, Li X, Qi J, et al. Serum MicroRNA-155 as a potential biomarker to track disease in breast cancer. PLoS ONE. (2012) 7:e47003. doi: 10.1371/journal.pone.0047003

52. Noman AS, Uddin M, Chowdhury AA, Nayeem MJ, Raihan Z, Rashid MI, et al. Serum sonic hedgehog (SHH) and interleukin-(IL-6) as dual prognostic biomarkers in progressive metastatic breast cancer. Sci Rep. (2017) 7:112. doi: 10.1038/s41598-017-01268-4

53. Ma X, Yuan T, Yang C, Wang Z, Zang Y, Wu L, et al. X-inactive-specific transcript of peripheral blood cells is regulated by exosomal Jpx and acts as a biomarker for female patients with hepatocellular carcinoma. Ther Adv Med Oncol. (2017) 9:665-77. doi: 10.1177/1758834017731052

54. Zhang K, Zhao S, Wang Q, Yang HS, Zhu J, Ma R. Identification of microRNAs in nipple discharge as potential diagnostic biomarkers for breast cancer. Ann Surg Oncol. (2015) 22:536-44. doi: 10.1245/s10434-015-4586-0

55. Ravelli A, Reuben JM, Lanza F, Anfossi S, Cappelletti MR, Zanotti L, et al. Breast cancer circulating biomarkers: advantages, drawbacks, and new insights. Tumor Biol. (2015) 36:6653-65. doi: 10.1007/s13277-015-3944-7

56. Katsuda T, Kosaka NOT. The roles of extracellular vesicles in cancer biology: toward the development of novel cancer biomarkers. Proteomics. (2014) 14:412-25. doi: 10.1002/pmic.201300389

57. Kleiveland CR. Peripheral blood mononuclear cells. In: Verhoeckx K, Cotter P, López-Expósito I, Kleiveland C, Lea T, Mackie A, editors. The Impact of Food Bioactives on Health. Cham: Springer (2015). p. 161-7.

58. Yao S, Kwan ML, Ergas IJ, Roh JM, Cheng TYD, Hong CC, et al. Association of serum level of Vitamin D at diagnosis with breast cancer survival a case-cohort analysis in the pathways study. JAMA Oncol. (2017) 3:3517. doi: 10.1001/jamaoncol.2016.4188

59. Bhan A, Hussain I, Ansari KI, Kasiri S, Bashyal A, Mandal SS Antisense transcript long noncoding RNA (lncRNA) HOTAIR is transcriptionally induced by estradiol. J Mol Biol. (2013) 425:3707-22. doi: 10.1016/j.jmb.2013.01.022

60. Russo J, Santucci-Pereira J, De Cicco RL, Sheriff F, Russo PA, Peri S, et al. Pregnancy-induced chromatin remodeling in the breast of postmenopausal women. Int J Cancer. (2012) 131:1059-70. doi: 10.1002/ijc.27323

61. Gayen S, Maclary E, Buttigieg E, Hinten M, Kalantry S. A primary role for the Tsix lncRNA in maintaining random X-chromosome inactivation. Cell Rep. (2015) 11:1251-65. doi: 10.1016/j.celrep.2015.04.039 
62. Navarro P, Chambers I, Karwacki-Neisius V, Chureau C, Morey C, Rougeulle C, et al. Molecular coupling of Xist regulation and pluripotency. Science. (2008) 321:1693-5. doi: 10.1126/science.1160952

63. Minkovsky A, Barakat TS, Sellami N, Chin MH, Gunhanlar N, Gribnau JPK. The pluripotency factor-bound intron 1 of Xist is dispensable for $\mathrm{X}$ chromosome inactivation and reactivation in vitro and in vivo. Cell Rep. (2013) 3:905-18. doi: 10.1016/j.celrep.2013.02.018

64. Pontier DB, Gribnau J. Xist regulation and function eXplored. Hum Genet. (2011) 130:223-36. doi: 10.1007/s00439-011-1008-7

65. Gontan C, Achame EM, Demmers J, Barakat TS, Rentmeester E, Van Ijcken W, et al. RNF12 initiates X-chromosome inactivation by targeting REX1 for degradation. Nature. (2012) 485:386-90. doi: 10.1038/nature 11070

66. Navarro P, Oldfield A, Legoupi J, Festuccia N, Dubois AS, Attia M, et al. Molecular coupling of Tsix regulation and pluripotency. Nature. (2010) 468:457-60. doi: 10.1038/nature09496

67. Son MY, Choi H, Han YM, Cho YS. Unveiling the critical role of REX1 in the regulation of human stem cell pluripotency. Stem Cells. (2013) 31:2374-87. doi: 10.1002/ stem.1509

68. Almozyan S, Colak D, Mansour F, Alaiya A, Al-Harazi O, Qattan A, et al. PD-L1 promotes OCT4 and Nanog expression in breast cancer stem cells by sustaining PI3K/AKT pathway activation. Int J Cancer. (2017) 141:140212. doi: $10.1002 / \mathrm{ijc} .30834$

Conflict of Interest: The authors declare that the research was conducted in the absence of any commercial or financial relationships that could be construed as a potential conflict of interest.

Copyright $\odot 2020$ Salama, Adbeltawab and El Tayebi. This is an open-access article distributed under the terms of the Creative Commons Attribution License (CC BY). The use, distribution or reproduction in other forums is permitted, provided the original author(s) and the copyright owner(s) are credited and that the original publication in this journal is cited, in accordance with accepted academic practice. No use, distribution or reproduction is permitted which does not comply with these terms. 